\title{
NONPARABOLIC SUBGROUPS OF THE MODULAR GROUP
}

\author{
by CAROL TRETKOFF
}

(Received 17 June, 1974; revised 13 March, 1975)

Introduction. In this paper we shall discuss maximal nonparabolic and maximal normal nonparabolic subgroups of the modular group $\Gamma=\left\langle\omega, \varphi ; \omega^{2}=\varphi^{3}=1\right\rangle$. The modular group may also be defined as the group of fractional linear transformations $w=(a z+b) /(c z+d)$, where $a, b, c, d$ are rational integers with $a d-b c=1$. Here, a maximal nonparabolic subgroup of $\Gamma$ is a subgroup that contains no parabolic elements and any proper subgroup of $\Gamma$ which contains $S$ contains parabolic elements. Similarly, a maximal normal nonparabolic subgroup is a normal nonparabolic subgroup of $\Gamma$ which is not contained in any larger normal nonparabolic subgroup of $\Gamma$.

In connection with maximal nonparabolic subgroups we are, in particular, interested in Neumann subgroups, where by a Neumann subgroup we mean a subgroup $N^{*}$ of the homogeneous modular group $\Gamma^{*}=S L(2, Z)$ which contains the matrix

$$
\omega=\left[\begin{array}{rr}
0 & 1 \\
-1 & 0
\end{array}\right] \text { and has the elements } \tau^{n}, \quad \tau=\left[\begin{array}{ll}
1 & 1 \\
0 & 1
\end{array}\right] \text {, }
$$

as right coset representatives in $\Gamma^{*}$. Magnus [7] and Neumann [13] show that Neumann subgroups $N^{*}$ of $\Gamma^{*}$ define maximal nonparabolic subgroups $N$ of $\Gamma$ which we will also call Neumann subgroups. Neumann found uncountably many Neumann subgroups and Magnus conjectured that Neumann's list was complete. In this paper we shall show that Neunann did not find all of them, and that, in fact, there exist Neumann subgroups with all possible subgroup structures except those whose free product decomposition contains a free group of odd rank; whether there exist Neumann subgroups whose free product structure contains a free group of odd rank is unknown.

In section two on normal nonparabolic subgroups, we show that if $G$ is a normal subgroup of finite index in $\Gamma$ and if the parabolic class number $t$ of $G$ is greater than 1 , then $G^{\prime}$, its commutator subgroup, contains only hyperbolic elements. By definition, if $n$ is the level of $G$ (the smallest integer $n$ such that $\tau^{n} \in G$ ), the parabolic class number is the number of distinct conjugates $u \tau^{n} u^{-1}$ in $G$ where $u \in \Gamma$. For all normal subgroups $G$ of genus zero with $t$ greater than 1, we find maximal normal nonparabolic subgroups containing $G^{\prime}$.

At this time I would like to thank Wilhelm Magnus and James Humphreys for their interest in this work; I would also like to thank the referee for his careful reading and helpful suggestions especially in correcting part of the proof of Theorem 2 .

1. Neumann subgroups. In 1932 B. H. Neumann [13] investigated subgroups $N^{*}$ of the homogeneous modular group $S L(2, Z)$ which are defined by the condition that, for any ordered pair of relatively prime integers $(a, c), N^{*}$ contains exactly one matrix in which the first column consists of the ordered pair $(a, c)$. Neumann investigated these subgroups in connection with a 
problem on the foundations of geometry while Magnus in 1973 investigated these groups as nonparabolic subgroups of the modular group. We have the following theorem.

THEOREM. A subgroup $\Lambda$ of the modular group $\Gamma$ is a maximal nonparabolic subgroup if either of the following two conditions is satisfied:

(i) The elements $\tau^{\lambda}=(\omega \varphi)^{\lambda}, \lambda=0,+1,+2, \ldots$ form a complete system of distinct right coset representatives of $\Lambda$ in $\Gamma$.

(ii) If $\Lambda^{*}$ is the homogeneous group generated by the $2 \times 2$ matrices defining the elements of $\Lambda$, then every ordered pair of relatively prime integers $(a, c)$ appears exactly once as the pair of elements in the first column of one of these matrices.

Condition (ii) implies (i) and (i) implies (ii) if

$$
\omega=\left[\begin{array}{rr}
0 & -1 \\
1 & 0
\end{array}\right] \text { or some other element } \gamma \text { with } \gamma^{n}=\left[\begin{array}{rr}
-1 & 0 \\
0 & -1
\end{array}\right] \text { is in } \Lambda \text {. }
$$

For a proof of this theorem see Magnus [8].

Using the Reidemeister-Schreier process for finding generators and relations for a subgroup, one can show that the problem of finding Neumann subgroups $N$, that is subgroups of $\Gamma$ satisfying the property (i) and containing $\omega$, is equivalent to finding a permutation $f$ of the integers which satisfies the following three conditions:

(i) $f(f(\lambda))=\lambda$,

(ii) $f(\lambda-1)=1+f(f(\lambda)+1)$,

(iii) $f(0)=0$.

The condition that $f(0)=0$ corresponds to the condition that $\omega \in N$. To see this we write

$$
\Gamma=\left\langle\omega, \tau ; \omega^{2}=(\omega \tau)^{3}=1\right\rangle .
$$

Then, using the $\tau^{\lambda}$ as coset representatives, $N$ is generated by

$$
\gamma_{\lambda}=\tau^{\lambda} \omega \overline{\left(\tau^{\lambda} \omega\right)^{-1}}=\tau^{\lambda} \omega \tau^{-f(\lambda)}
$$

where $\tau^{f(\lambda)}$ is the right coset representative of $\tau^{\lambda} \omega$. It follows immediately that if $\omega \in N$ we must have $f(0)=0$. The relations are

$$
\gamma_{\lambda} \gamma_{f(\lambda)}=1 \text { and } \gamma_{\lambda-1}^{-1} \gamma_{\lambda} \gamma_{f(\lambda)+1}=1
$$

Note that different permutations of the integers $f: \mathbf{Z} \rightarrow \mathbf{Z}$ satisfying conditions (i), (ii), and (iii) define different Neumann subgroups since the generators are

$$
\gamma_{\lambda}=\left[\begin{array}{cc}
\lambda & -1-\lambda f(\lambda) \\
1 & -f(\lambda)
\end{array}\right]
$$

and there cannot be two different matrices with first column $(\lambda, 1)$ in the same Neumann subgroup. The uncountably many Neumann subgroups found by B. H. Neumann are described in the following theorem $[8,13]$. 
NeumanN's Theorem. Let $h_{l}, l=1,2,3, \ldots$ be an infinite sequence in which each $h_{l}$ is either 0 or 1. Let $k_{l}=1-h_{l}$ and define $g_{l}$ by

$$
g_{1}=2, \quad g_{l}=6 l-5 \sum_{i=1}^{l-1} h_{i}-4 \quad(l \geqq 2) .
$$

Then $g_{l+1}-g_{l}=1$ if $h_{l}=1$ and $g_{l+1}-g_{l}=6$ if $h_{l}=0$. Every integer $\geqq 2$ can be represented in exactly one manner as an expression of one of the forms

$$
g_{l},\left(g_{l}+\sigma\right) k_{l} \quad(\sigma=1,2,3,4,5) .
$$

The permutations of the integers defined by the table below satisfy conditions (i), (ii), and (iii).

\begin{tabular}{l|l}
\multicolumn{1}{c|}{$\lambda$} & \multicolumn{1}{c}{$f(\lambda)$} \\
\hline$-3 l-1$ & $(-3 l) h_{l}+\left(g_{l}+3\right) k_{l}$ \\
$-3 l$ & $(-3 l-1) h_{l}+\left(g_{l}+1\right) k_{l}$ \\
$1-3 l$ & $g_{l}-1$ \\
-1 & -1 \\
0 & 0 \\
1 & -2 \\
$g_{l}$ & $(-3 l-2) h_{l}+g_{l} k_{l}$ \\
$\left(g_{l}+1\right) k_{l}$ & $-3 l k_{l}$ \\
$\left(g_{l}+2\right) k_{l}$ & $\left(g_{l}+2\right) k_{l}$ \\
$\left(g_{l}+3\right) k_{l}$ & $(-3 l-1) k_{l}$ \\
$\left(g_{l}+4\right) k_{l}$ & $\left(g_{l}+4\right) k_{l}$ \\
$\left(g_{l}+5\right) k_{l}$ & $(-3 l-2) k_{l}$
\end{tabular}

We start our study of Neumann subgroups by asking whether they are infinitely generated and whether they are first kind. In order to define what it means for a Fuchsian group to be first kind, we must first define a limit point of a Fuchsian group $F$ to be a limit of a sequence of images $V_{n} z, V_{n} \in F$. The limit set $L$ of $F$, that is the set of all limit points of $F$, is either the real line or else a perfect nowhere dense subset of the real line [5]. If the limit set is the entire line, $F$ is called first kind or horocyclic. Otherwise $F$ is called second kind.

THEOREM 1. All Neumann subgroups of the modular group are infinitely generated and first kind.

Proof. By a theorem of Marden [9], we know that a Fuchsian group $G$ is finitely generated if and only if every fundamental region has a finite number of sides. It is well known that a fundamental region $\mathbf{R}$ for the modular group is $\left\{z:-\frac{1}{2}<\operatorname{Re} z \leqq \frac{1}{2},|z|>1\right\}$. Thus, since the fundamental region for a Neumann subgroup constructed by taking the union of the regions $\tau^{n} \mathbf{R}$ has infinitely many sides, a Neumann subgroup must be infinitely generated. 
We shall now show that for any Neumann subgroup $N$,

$$
L_{\infty}=\left\{\alpha \mid \alpha=\lim _{n \rightarrow \infty} V_{n}(\infty) \text { where } V_{n} \in N\right\}
$$

is the real line and hence $N$ is first kind. Let $x$ be any element on the real line and let $a_{n} / c_{n}$ be a sequence of distinct rationals with $\left(a_{n}, c_{n}\right)=1$ such that $\lim _{n \rightarrow \infty} a_{n} / c_{n}=x$. Then by the first theorem stated in this section, we know that there exists a transformation $V_{n} \in N$ defined by

$$
V_{n}(z)=\frac{a_{n} z+b_{n}}{c_{n} z+d_{n}}
$$

in $N$. Now $V_{n}(\infty)=a_{n} / c_{n}$ so $\lim _{n \rightarrow \infty} V_{n}(\infty)=x$. Thus $L_{\infty}=\mathbf{R}$ and Neumann subgroups are first kind.

Since the modular group is a free product of the cyclic group $\sigma_{2}$ of order 2 and the cyclic group $\sigma_{3}$ of order 3, we know from the Kurosh subgroup theorem that structurally a Neumann subgroup must be a free product of groups isomorphic to $\mathbf{Z}, \sigma_{2}$ and $\sigma_{3}$, where at least one of these types appears infinitely many times and $\sigma_{2}$ appears at least once since Neumann subgroups contain $\omega$. We have the following theorem.

THEOREM 2. There exist Neumann subgroups with all possible structures with the exception that, when the free factor is finitely generated, its rank is even. (It may be true that all possible structures are possible.)

Proof. To prove this theorem we shall construct permutations $f: \mathbf{Z} \rightarrow \mathbf{Z}$ satisfying the three conditions $f(0)=0, f(f(\lambda))=\lambda$, and $f(\lambda-1)=1+f(f(\lambda)+1)$. For factors of the free product that are isomorphic to $\sigma_{2}$ we use the following pattern.

Pattern 1:

\begin{tabular}{l|l}
\multicolumn{1}{c|}{$\lambda$} & $f(\lambda)$ \\
\hline$k-1$ & $l$ \\
$k$ & $k$ \\
$k+1$ & $l-1$
\end{tabular}

Here and in the following patterns $l$ is the smallest integer $<0$ that has been already assigned and $k$ is the first positive integer that has not yet been assigned. We use pattern 2 for free factors that are isomorphic to $\sigma_{3}$ and pattern 3 for free factors that are isomorphic to $F_{2}$, the free group of rank 2.

Pattern 2:

\begin{tabular}{l|l}
\multicolumn{1}{c|}{$\lambda$} & $f(\lambda)$ \\
\hline$k-1$ & $l$ \\
$k$ & $k+1$ \\
$k+1$ & $k$ \\
$k+2$ & $l-1$
\end{tabular}


Pattern 3:

\begin{tabular}{l|l}
\multicolumn{1}{c|}{$\lambda$} & $f(\lambda)$ \\
\hline$k-1$ & $l$ \\
$k$ & $k+4$ \\
$k+1$ & $k+6$ \\
$k+2$ & $l-2$ \\
$k+3$ & $k+7$ \\
$k+4$ & $k$ \\
$k+5$ & $l-1$ \\
$k+6$ & $k+1$ \\
$k+7$ & $k+3$ \\
$k+8$ & $l-3$
\end{tabular}

Note that each of these patterns can be attached consecutively in any order since they all end by sending the greatest positive integer assigned to the least negative integer already assigned, and this just becomes the beginning of the next pattern to be used in constructing the permutation $f$.

To prove that these patterns give the desired results we must use the relations

$$
\gamma_{\lambda-1}=\gamma_{\lambda} \gamma_{S(\lambda)+1} \text { and } \gamma_{\lambda} \gamma_{f(\lambda)}=1
$$

to write the group as a free product. For pattern 1, we have generators

$$
\gamma_{k-1}, \gamma_{k}, \gamma_{k+1}
$$

and the following relations.

$$
\begin{aligned}
& \gamma_{k-1}=\gamma_{k} \gamma_{f(k)+1} \text { or } \gamma_{k-1}=\gamma_{k} \gamma_{k+1} \\
& \gamma_{k}=\gamma_{k+1} \gamma_{f(k+1)+1} \text { or } \gamma_{k}=\gamma_{k+1} \gamma_{l} \text { or } \gamma_{k-1}=\gamma_{k} \gamma_{k+1} \\
& \gamma_{k} \gamma_{k}=1
\end{aligned}
$$

So, if $\gamma_{k-1}$ is taken into account in the previous pattern, we can write

$$
\gamma_{k+1}=\gamma_{k}^{-1} \gamma_{k-1}
$$

and hence we are left with generator $\gamma_{k}$ and the relation $\gamma_{k}^{2}=1$.

For pattern 2, we have generators

$$
\gamma_{k-1}, \gamma_{k}, \gamma_{k+1}, \gamma_{k+2}
$$

and relations

(1) $\gamma_{k-1}=\gamma_{k} \gamma_{k+2}$

(2) $\gamma_{k}=\gamma_{k+1} \gamma_{k+1}$

(3) $\gamma_{k+1}=\gamma_{k+2} \gamma_{l}$ or $\gamma_{k-1}=\gamma_{k} \gamma_{k+2}$

(4) $\gamma_{k}=\gamma_{k+1}^{-1}$ 
Now, relations (2) and (4) imply that $\gamma_{k}^{3}=1$, and relations (1) and (3) imply that if we know $\gamma_{k-1}$ we can eliminate $\gamma_{k+2}$. Thus we have one generator $\gamma_{k}$ and one relation $\gamma_{k}^{3}=1$.

Similarly, for pattern 3 , we have generators

and relations

$$
\gamma_{k-1}, \ldots, \gamma_{k+8}
$$
(1) $\gamma_{k-1}=\gamma_{k} \gamma_{k+s}$
(7) $\gamma_{k+5}=\gamma_{k+6} \gamma_{k+2}$
(2) $\gamma_{k}=\gamma_{k+1} \gamma_{k+7}$
(8) $\gamma_{k+6}=\gamma_{k+7} \gamma_{k+4}$
(3) $\gamma_{k+1}=\gamma_{k+2} \gamma_{l-1}$
(9) $\gamma_{k+7}=\gamma_{k+8} \gamma_{l-2}$
(4) $\gamma_{k+2}=\gamma_{k+3} \gamma_{k+8}$
(10) $\gamma_{k} \gamma_{k+4}=1$
(5) $\gamma_{k+3}=\gamma_{k+4} \gamma_{k+1}$
(11) $\gamma_{k+1} \gamma_{k+6}=1$
(6) $\gamma_{k+4}=\gamma_{k+5} \gamma_{l}$
(12) $\gamma_{k+3} \gamma_{k+7}=1$

Using relations (10), (11), and (12), we can remove $\gamma_{k+4}, \gamma_{k+6}$, and $\gamma_{k+7}$ from the list of generators and get
(1) $\gamma_{k-1}=\gamma_{k} \gamma_{k+5}$
(2) $\gamma_{k}=\gamma_{k+1} \gamma_{k+3}^{-1}$
(6) $\gamma_{k}^{-1}=\gamma_{k+5} \gamma_{k-1}^{-1}$
(3) $\gamma_{k+1}=\gamma_{k+2} \gamma_{k+5}^{-1}$
(7) $\gamma_{k+5}=\gamma_{k+1}^{-1} \gamma_{k+2}$
(4) $\gamma_{k+2}=\gamma_{k+3} \gamma_{k+8}$
(8) $\gamma_{k+1}^{-1}=\gamma_{k+3}^{-1} \gamma_{k}^{-1}$
(5) $\gamma_{k+3}=\gamma_{k}^{-1} \gamma_{k+1}$
(9) $\gamma_{k+3}^{-1}=\gamma_{k+8} \gamma_{k+2}^{-1}$

Now, relations (2), (5) and (8), (1) and (6), (4) and (9), and (3) and (7) are the same, so we need only deal with relations (1), (2), (3) and (4). Given $\gamma_{k-1}$ from the previous set of generators and keeping $\gamma_{k}$ and $\gamma_{k+1}$, we can remove $\gamma_{k+3}, \gamma_{k+5}$ and $\gamma_{k+8}$ from the list of generators and at the same time remove relations (1), .., (4). Hence we are left with generators $\gamma_{k}$ and $\gamma_{k+1}$ and no relations.

The question that remains is how do we begin the permutation. We want to show that if $r+s+t=\infty$ where $r, s$, and $t$ are non-negative integers, that we can construct a Neumann subgroup whose free product structure has $r$ factors isomorphic to $\sigma_{2}, s$ factors isomorphic to $\sigma_{3}$ and $t$ factors isomorphic to the free group of rank 2. Recall that $r$ must be at least one since $\omega$ must be in the Neumann subgroup and $\omega^{2}=1$. There are three cases to consider: $r \geqq 2$, $r=1$ and $s \geqq 1$, and $r=1$ and $s=0$. If $r \geqq 2$, begin the permutation $f$ with pattern 1 with $k=1, l=0$. This contributes $\sigma_{2} * \sigma_{2}$ to the free product structure. Then complete the permutation by using pattern $1 r-2$ times, pattern $2 s$ times and pattern $3 t$ times. However, if $r=1$ and $s \geqq 1$, begin with pattern 2 with $k=1, l=0$ and if $r=1$ and $s=0$, begin with pattern 3 with $k=1, l=0$. It is easy to check that these beginnings contribute $\sigma_{2} * \sigma_{3}$ and $\sigma_{2} * F_{2}$ to the free product structure. Then one can complete the permutation $f$ by using patterns 2 and 3 the appropriate number of times.

The Neumann subgroups that Neumann found are in one to one correspondence with infinite sequences of zeros and ones. It turns out that each zero contributes the free product of three cyclic groups of order 2 to the free product structure of the Neumann subgroup, while each one contributes a cyclic group of order 3 . 
To see this, we first note that if $h_{l}=0$ and $l \geqq 2$, then

\begin{tabular}{l|l}
\multicolumn{1}{c|}{$\lambda$} & \multicolumn{1}{c}{$f(\lambda)$} \\
\hline$-3 l-2$ & $g_{l}+5$ \\
$-3 l-1$ & $g_{l}+3$ \\
$-3 l$ & $g_{l}+1$ \\
$-3 l+1$ & $g_{l}-1$ \\
\multicolumn{1}{c}{} & $\vdots$ \\
$g_{l}-1$ & $-3 l+1$ \\
$g_{l}$ & $g_{l}$ \\
$g_{l}+1$ & $-3 l$ \\
$g_{l}+2$ & $g_{l}+2$ \\
$g_{l}+3$ & $-3 l-1$ \\
$g_{l}+4$ & $g_{l}+4$ \\
$g_{l}+5$ & $-3 l-2$
\end{tabular}

Here the integers between $-3 l$ and $g_{l}$ have been previously assigned. By comparing this with pattern 1, we note that this portion of Neumann's permutation consists of pattern 1 repeated three times. Thus it must correspond to the free product of three cyclic groups of order 2.

Now if $h_{l}=1$ and $l \geqq 2$, then

\begin{tabular}{l|l}
\multicolumn{1}{c|}{$\lambda$} & $f(\lambda)$ \\
\hline$-3 l-2$ & $g_{l}$ \\
$-3 l-1$ & $-3 l$ \\
$-3 l$ & $-3 l-1$ \\
$-3 l+1$ & $g_{l}-1$ \\
$\vdots$ & $\vdots$ \\
$g_{l}-1$ & $-3 l+1$ \\
$g_{l}$ & $-3 l-2$
\end{tabular}

where the integers between $-3 l$ and $g_{l}$ have been previously assigned. Comparing this with pattern 2, one finds that this portion of the permutation is similar to pattern 2 except for the fact that the role of the negative and positive integers has been reversed.

It remains to check the portion of Neumann's permutation around zero (which is unchanged for all sequences of zeros and ones). Here

\begin{tabular}{r|r}
\multicolumn{1}{c|}{$\lambda$} & $f(\lambda)$ \\
\hline-2 & 1 \\
-1 & -1 \\
0 & 0 \\
1 & -2
\end{tabular}


It is easy to check using relations $\gamma_{\lambda} \gamma_{f(\lambda)}=1$ and $\gamma_{\lambda-1}^{-1} \gamma_{\lambda} \gamma_{f(\lambda)+1}=1$ that this portion of the permutation contributes a free product of two cyclic groups of order 2 generated by $\gamma_{-1}$ and $\gamma_{0}$ to the structure of the Neumann subgroup.

Thus the free product structure of Neumann's Neumann subgroups contain no free factors. This implies that Magnus's conjecture [8] that Neumann's list is complete is false.

2. Normal nonparabolic subgroups of $\boldsymbol{\Gamma}$. We now study the normal nonparabolic subgroups of the modular group $\Gamma=\left\langle\omega, \varphi ; \omega^{2}=\varphi^{3}=1\right\rangle$ and consider the problem of finding maximal ones. We start by proving the following theorem.

THEOREM 3. The normal nonparabolic subgroups $N$ of $\Gamma$ are all free of infinite rank.

Proof. From Newman [15] we know that every normal subgroup of $\Gamma$ except $\Delta(\omega)$ and $\Delta(\varphi)$ is free, where by $\Delta(x)$ we mean the normal closure of $x$ in $\Gamma$. Certainly, a nonparabolic subgroup of $\Gamma$ must have infinite index in $\Gamma$ since none of the elements $\tau^{n}, n \neq 0$, is in $\Gamma$, where $\tau=\omega \varphi=\left[\begin{array}{ll}1 & 1 \\ 0 & 1\end{array}\right]$. That the normal nonparabolic subgroups of $\Gamma$ have infinite rank then follows from B. Baumslag's theorem that if a subgroup $S$ of infinite index in a free product of two groups $P$ contains a normal subgroup of $P$, then $S$ must be of infinite rank [1].

We shall need the following lemma, which is due to Mason [10].

LEMMA. If $G$ is a normal subgroup of finite index in the modular group $\neq \Delta(\omega), \Delta(\varphi)$ or $\Gamma^{\prime}$, then $G^{\prime}$, the commutator subgroup, is normal in $\Gamma$ and contains only hyperbolic elements.

Magnus [7] shows that $\Gamma(2)^{\prime}$ is maximal normal hyperbolic by proving that $\Gamma / \Gamma(2)^{\prime}$ is just-infinite, where by a just-infinite group we mean one in which every (non-trivial) normal subgroup has finite index [12]. Here, by $\Gamma(n)$ we mean the principal congruence subgroup of $\Gamma$ of level $n$, that is the set of linear fractional transformations $\frac{a z+b}{a z+b}$ with

$$
a \equiv d \equiv+1 \bmod n \quad \text { and } \quad \dot{b} \equiv c \equiv 0 \bmod n .
$$

The fact that $\Gamma(2)^{\prime}$ is maximal normal hyperbolic then follows from the correspondence theorem and the fact that all subgroups of finite index in $\Gamma$ must contain parabolic elements. We shall show that $\Gamma(3)^{\prime}$ is also maximal normal hyperbolic using some results from Mason's paper [11] and some facts about space groups.

We know that if $G$ is a normal subgroup of $\Gamma \neq \Delta(\omega)$ or $\Delta(\varphi), G$ has the following presentation

$$
G=\left\langle P_{1}, P_{2}, \ldots, P_{t}, A_{1}, B_{1}, \ldots, A_{g}, B_{g} ; P_{1} \ldots P_{t} \Pi\left[A_{i}, B_{i}\right]=+I\right\rangle,
$$

where the set $\left\{P_{1}, \ldots, P_{t}\right\}$ has the following properties:

(i) every parabolic element of $G$ is conjugate over $G$ to some power of a $P_{i}(1 \leqq i \leqq t)$

(ii) no non-trivial power of a $P_{i}$ is conjugate over $G$ to a power of $P_{i}(1 \leqq i, j \leqq t, i \neq j)$ (Lehner [4]).

Now, as in Mason [11], we define a permutational representation $\theta$ of $\Gamma$ on the $t$ symbols 
$P_{1}, \ldots, P_{r}$. Let $X \in \Gamma$. Then $X P_{i} X^{-1}=X_{j} P_{j} X_{j}^{-1}$ for some $X_{j} \in G$, by the properties of the set $\left\{P_{1}, \ldots, P_{t}\right\}$ stated earlier, and $\Pi_{X}\left(P_{i}\right)=P_{j}$ is a permutation of the set $\Omega=\left\{P_{1}, \ldots, P_{t}\right\}$. Mason proves the following theorem [11].

MASON'S THEOREM. Let $S_{t}$ be the symmetric group on $t$ symbols. The map $\theta: \Gamma \rightarrow S_{t}$ defined by $\theta(X)=\Pi_{X}$ for all $X \in \Gamma$ is a homomorphism from $\Gamma$ onto a transitive subgroup of $S_{t}$. The kernel of $\theta$ equals $G^{*}$ and the stabilizer in $\theta(\Gamma)$ of $P_{1} \in \Omega$ is $\theta\left(G^{*}\right)$, so that all the stabilizers in $\theta(\Gamma)$ of the elements of $\Omega$ are cyclic groups of fixed order $k$.

Here $G^{\tau}$ is the group generated by $\tau$ and $G$ where $\tau=\omega \varphi$ and $G^{*}$ is the group generated by $\tau^{k}$ and $G$, where $1 \leqq k \leqq t$ and $k$ is the least integer such that

$$
\omega \tau^{k} \equiv \tau^{k} \omega(\bmod G)
$$

From this theorem it follows that when $G$ is maximal normal, that is, when $\Gamma / G$ is simple, $\operatorname{ker} \theta=G$ and $\theta$ gives a faithful permutational representation of $\Gamma / G$. Also, Mason has remarked in $\left[11\right.$, p. 456] that when $G=\Gamma(N)$, then $G^{*}=\operatorname{ker} \theta=G$. In particular, this tells us that when $G=\Gamma(3), \operatorname{ker} \theta=\Gamma(3)$ and $\theta$ gives a permutational representation of $\Gamma / \Gamma(3) \cong A_{4}$. We shall need this fact in order to show that $\Gamma / \Gamma(3)^{\prime}$ is a space group and $\Gamma(3)^{\prime}$ is maximal normal hyperbolic.

We may define a space group $S$ of dimension $n$ to be an extension of a free abelian group $A$ of finite rank $n$ by a finite group $B$ such that the centralizer of $A$ in $S$ is $A$. This is equivalent to saying that an $n$-dimensional space group is an extension of a free abelian group of rank $n$ by a finite group $B$ using a monomorphism $\rho: B \rightarrow$ Aut $A$. The finite group $B$ is called the point group. Here the monomorphism $\rho$ is just conjugation of the elements of $A$ by the elements of $B$. Hence the group $\Gamma / G^{\prime}$ is a space group with free abelian normal subgroup $G / G^{\prime}$ and point group $\Gamma / G$ whenever $\operatorname{ker} \theta=G$. In particular, $\Gamma / G^{\prime}$ is a space group when $\Gamma / G$ is simple, $G \neq \Delta(\omega)$ or $\Delta(\varphi)$, and when $G=\Gamma(3)$.

Now the only free normal subgroups of genus zero of the modular group are $\Gamma(2), \Gamma(3)$, $\Gamma(4)$ and $\Gamma(5)$. This follows from a simple calculation using the formula for the genus of a subgroup of the modular group [14]. And, without additional information about the normal closure in $\Gamma$ of the generators $A_{1}, B_{1}, \ldots, A_{g}, B_{g}$ for a normal subgroup $G$ of genus $g$, we cannot obtain maximal normal hyperbolic subgroups containing $G^{\prime}$. We do know that the normal closure of $A_{1}, B_{1}, \ldots, A_{g}, B_{g}$ in $G$ is hyperbolic since any parabolic element in $G$ must be conjugate to some $P_{i}, 1 \leqq i \leqq t$, and conjugates of $P_{i}$ are not in the normal closure of $A_{1}, B_{1}, \ldots, A_{g}, B_{g}$. Hence, if the normal closure of $A_{1}, B_{1}, \ldots, A_{g}, B_{g}$ in $G$ is normal in $\Gamma$, we would have a start in determining maximal normal hyperbolic subgroups containing $G^{\prime}$ when $G$ has genus greater than zero. Now $\Gamma(4)^{\prime}$ is certainly not maximal normal hyperbolic since it is contained in $\Gamma(2)^{\prime}$, and while $\Gamma(5)^{\prime}$ is not maximal normal hyperbolic, we can find a maximal normal hyperbolic subgroup containing $\Gamma(5)^{\prime}$. First, however, we shall show that $\Gamma(3)^{\prime}$ is maximal normal nonparabolic.

Mason [11] shows that there are no normal subgroups $N$ with infinite index in $\Gamma$ between $\Gamma(3)$ and $\Gamma(3)^{\prime}$ other than $\Gamma(3)^{\prime}$. Hence, using the following lemma, we have

THEOREM 4. $\Gamma / \Gamma(3)^{\prime}$ is just infinite, so that $\Gamma(3)^{\prime}$ is maximal normal hyperbolic. 
LEMMA. A space group $S$ with maximal normal abelian subgroup $A$ and point group $B$ is just-infinite if and only if all normal subgroups $N$ of $S$ such that $N \subset A$ have finite index in $S$.

Proof. It follows from the definition of a just-infinite group that if $S$ is just-infinite, then $N \triangleleft S$ implies that $N$ has finite index.

Now assume that $S$ is not just-infinite and all normal subgroups $N \triangleleft S$ such that $N \subset A$ have finite index in $S$. Then there exists a subgroup $N^{*} \triangleleft S$ such that $\left[S: N^{*}\right]=\infty$. Since all normal subgroups of $S$ contained in $A$ have finite index, $N^{*} \cap A=1$. But this is impossible since if $N^{*} \cap A=1$, then $\left[N^{*}, A\right]=\left\{n a n^{-1} a^{-1} \mid n \in N^{*}, a \in A\right\}=1$, and this implies that $N^{*}$ is contained in the centralizer of $A$ in $S$. Since the centralizer of $A$ in $S$ is $A, N^{*}=1$.

In order to work with $\Gamma(5)^{\prime}$ we need the following theorem of McCarthy [12].

MCCARTHY'S THEOREM. If $G$ is a just-infinite group where $A$ is the maximal normal abelian subgroup that is free of positive rank $r$ and $B$ is the point group, then the monomorphism $\rho: B \rightarrow$ Aut $A$ affords a faithful $\mathbf{Z}$-irreducible representation of $B$ in $G L(r, \mathbf{Z})$. Conversely, let $B$ be a finite group which has a faithful Z-irreducible representation $\rho: B \rightarrow$ Aut $A$ and let $G$ be any extension of $A$ by $B$ using $\rho$; then $G$ is just-infinite.

We know that $\Gamma(5)$ is generated by $P_{1}, \ldots, P_{12}$, and Mason [11] shows that $\theta: \Gamma \rightarrow S_{12}$ is not primitive. Mason also shows that if $P_{1}, \ldots, P_{12}$ are properly arranged so that the blocks of imprimitivity are $\left\{P_{1}, P_{2}\right\},\left\{P_{3}, P_{4}\right\}, \ldots,\left\{P_{11}, P_{12}\right\}$, then the group $L$ generated by

$$
\left\{P_{1} P_{2}, P_{3} P_{4}, \ldots, P_{11} P_{12} \text { and } G^{\prime}\right\}
$$

is normal in $\Gamma$ and is hyperbolic. This means that the permutation representation of $A_{5}$ of degree 12 obtained by conjugating $P_{1}, \ldots, P_{12}$ by the elements of $\Gamma / \Gamma(5)$ is reducible over the integers. In particular, with basis elements $A_{1}=P_{1} P_{2}, A_{2}=P_{3} P_{4}, A_{3}=P_{5} P_{6}, A_{4}=P_{7} P_{8}$, $A_{5}=P_{9} P_{10}, A_{6}=P_{11} P_{12}, P_{1}, P_{3}, P_{5}, P_{6}, P_{9}$, and $P_{11}$, we see that the representation is reducible into two 6 degree representations of $A_{5}$, the first $\theta_{1}$ being a transitive permutation representation on the blocks $\left\{P_{1}, P_{2}\right\}, \ldots,\left\{P_{11}, P_{12}\right\}$ while the second $\theta_{2}$ is an integral representation with 1 's and -1 's as entries. Now, the representation on $A_{1}, A_{2}, \ldots, A_{6}$ is doubly transitive since by Mason's Theorem the stabilizer of $P_{1}$ is a 5-cycle which must be transitive on $A_{2}, \ldots, A_{6}$. Here we are using the lemma that if $G$ is a transitive permutation group on $\Omega$, then $G$ is $k$-fold transitive if and only if any $G_{\alpha}$ is $k-1$ fold transitive on $\Omega-\{\alpha\}$, where $G_{\alpha}$ is the stabilizer of $\alpha \in \Omega$.

\begin{tabular}{l|rrrcc}
\multicolumn{1}{c}{} & $\mathscr{C}_{1}$ & $\mathscr{C}_{2}$ & $\mathscr{C}_{3}$ & $\mathscr{C}_{4}$ & $\mathscr{C}_{5}$ \\
\cline { 2 - 6 }$\chi_{1}$ & 1 & 1 & 1 & 1 & 1 \\
$\chi_{2}$ & 3 & -1 & 0 & $\frac{1+\sqrt{5}}{2}$ & $\frac{1-\sqrt{5}}{2}$ \\
$\chi_{3}$ & 3 & -1 & 0 & $\frac{1-\sqrt{5}}{2}$ & $\frac{1+\sqrt{5}}{2}$ \\
$\chi_{4}$ & 4 & 0 & 1 & -1 & -1 \\
$\chi_{5}$ & 5 & 1 & -1 & 0 & 0
\end{tabular}


By [2, p. 58] $\theta_{1}$, being doubly transitive, reduces over its splitting field into the identity representation and an absolutely irreducible representation. However $[2$, p. 57] $\theta$, being a transitive permutation representation, contains the identity representation only once over its splitting field. The character table of $A_{5}$ is as shown above.

It follows that $\theta_{2}$, if reducible over the integers, must split into two representations of degree 3 . Since the degree 3 representations have irrational characters, we see that $\theta_{2}$ must be irreducible over $\mathbf{Z}$. Hence we have the following theorem.

THEOREM 5. The group $L$, as defined by Mason, is a maximal normal hyperbolic subgroup of $\Gamma$ and $\Gamma / L$ is a just-infinite group with point group $A_{5}$ and a normal free abelian subgroup of rank 6.

We remark that Mason's Theorems that there are no subgroups of infinite index in $\Gamma$ between $\Gamma(2)^{\prime}$ and $\Gamma(2)$ and between $\Gamma(3)^{\prime}$ and $\Gamma(3)$ can also be proved using McCarthy's Theorem and the fact that the permutational representations $\theta$ defined by Mason's Theorem of $\Gamma / \Gamma(2) \cong S_{3}$ and $\Gamma / \Gamma(3) \cong A_{4}$ are doubly transitive on $t=3$ and $t=4$ symbols respectively, where $t$ is the parabolic class number. Since a doubly transitive representation reduces over its splitting field into the identity representation and an absolutely irreducible representation [2, p. 58], McCarthy's Theorem applies.

\section{REFERENCES}

1. B. Baumslag, Intersection of finitely generated subgroups in free products, J. London Math. Soc. 41 (1966), 673-679.

2. L. Dornhoff, Group representation theory, Part A, Marcel Dekker, Inc. (New York, 1971).

3. R. C. Gunning, Lecture on modular forms, Princeton University Press (Princeton, New Jersey, 1962). R.I., 1964 .

4. J. Lehner, Discontinuous groups and automorphic functions, Amer. Math. Soc., Providence, 1966).

5. J. Lehner, $A$ short course in automorphic functions, Holt, Rinehart and Winston (New York,

6. R. Lyndon, Two notes on Rankin's book on the modular group, J. of the Australian Math. Soc. (1973), 454-457.

7. W. Magnus, Rational representations of Fuchsian groups and nonparabolic subgroups of the modular group, Nachrichten der Akademie der Wissenschaften in Gottingen, MathematischPhysikalische Klasse, 1973.

8. W. Magnus, Non-Euclidean tesselations and their groups, Academic Press (1974). 85.

9. A. Marden, On finitely generated Fuchsian groups, Comment. Math. Helv. 42 (1967), 81$106-115$

10. A. W. Mason, Lattice subgroups of free congruence groups, Glasgow Math. J. 10 (1969),

11. A. W. Mason, Lattice subgroups of normal subgroups of genus zero of the modular group, Proc. London Math. Soc., XXIV (1972), 449-469.

12. D. McCarthy, Infinite groups whose proper quotient groups are finite, Communications in Pure and Applied Mathematics 21 (1968), 545-562.

13. B. H. Neumann, Uber ein gruppentheoretisch-arithmetisches Problem, Sitzungsber. Preuss. Akad. Wiss. Phys. Math. Kl., No. X (1933).

$\mathbf{G}$ 
14. M. Newman, Classification of normal subgroups of the modular group, Trans. American Math. Soc. 16 (1965), 267-277.

15. M. Newman, Integral matrices, Academic Press (New York, 1972).

16. R. A. Rankin, The modular group and its subgroups, The Ramanujan Institute (Madras, 1969).

Courant Institute of Mathematical Sciences

251 MERCER STREET

NEW YORK, NEW YORK 10012 